Vietnam Journal of Mechanics, VAST, Vol.39, No. 4 (2017), pp. 375-386

DOI:10.15625/0866-7136/11026

\title{
PHASE TRANSFORMATION SURFACES AROUND A CRACK TIP FOR A SHAPE MEMORY ALLOYS
}

\author{
Christian Lexcellent \\ Département de Mécanique Appliquée, FEMTO-st, Besancon Cedex, France \\ E-mail: christian.lexcellent@univ-fcomte.fr \\ Received February 21, 2017
}

\begin{abstract}
Depending of the shape of the crack tip e.g. with or without curvature, the size of the phase transformation surface between a mother phase A (austenite) and a producted phase $\mathrm{M}$ (martensite) is different. The presentation is focussed to the modes I and II (opening and shearing modes). The elastic stress field around the crack tip without curvature is known in the litterature and the use of "Linear Elastic Mechanical Theory" is consistent with the deformations amplitude associated the beginning of the phase transformation $(\mathrm{A} \Rightarrow \mathrm{M})$. In order to take into account the curvature at the crack tip, one uses the approximated expressions of Creager and Paris (1967). A special attention is devoted to take into account the asymmetry between tension and compression behavior in the surfaces prediction.
\end{abstract}

Keywords: Crack detection, damage detection, multi-cracks detection, stiffness method, element stiffness, element stiffness index distribution.

\section{INTRODUCTION}

Shape Memory Alloys (SMA) are potential materials for use in smart structures, actuators, medical devices and aeronautical materials. This is because of the very large recoverable strains (on the order of $8 \%$ for equi-atomic Ni-Ti) associated with their superelastic or pseudoelastic behavior. The extended use of SMA elements which are sometimes subjected to rather complex loading, raises the issue of service life of the systems and leads us to investigate SMA fracture and/or fatigue damage. As discussed by Daly et al. [1], fatigue and fracture behaviours and their possible consequences on "patients health" are of great concern in medical industry where Ni-Ti is widely used for medicals devices like stents.

For instance, Yi and Gao [2] investigated numerically the SMA fracture toughening mechanism under mode I loading and showed that the martensitic phase transformation (MPT) increases the toughness and decreases the crack tip intensity factor. Wang et al. [3] examined the stress induced martensite near the crack tip of a CT specimen by a FEM calculation.

(C) 2017 Vietnam Academy of Science and Technology 
Moreover, we can cite experimental work about the fracture behavior of $\mathrm{Ni}-\mathrm{Ti}$ tubes [4]. Robertson and Ritchie measured in-situ three dimensional strains, phases and crystallographic alignment ahead of a growing fatigue crack (after 100 cycles). Their measurements reveal that the majority of austenite grains were subjected to only $0.5-$ $1.0 \%$ elastic strain despite a macroscopic superelastic strain of $6-8 \%$ recovery associated with the MPT.

Daly et al. [1] performed a tensile test on an edge crack specimen of austenitic NiTi for a tip with curvature, using an in-situ optical technique to examine the shape and the size of the phase transformation zones. They measured the fracture toughness K1c at room temperature for fine grained polycrystalline nitinol sheets.

\section{PROBLEM WITH A CRACK TIP TO SOLVE}

\subsection{Stress field around the crack tip}

Let the tensor $\sigma(M(r, \theta))$ expressed in Cartesian coordinates

$$
\sigma(M(r, \theta))=\left(\begin{array}{ccc}
\sigma_{x x} & \sigma_{x y} & \sigma_{x z} \\
\sigma_{y x} & \sigma_{y y} & \sigma_{y z} \\
\sigma_{z x} & \sigma_{z y} & \sigma_{z z}
\end{array}\right) .
$$

Remember that

$$
\begin{aligned}
& \sigma_{y z}=\sigma_{z x}=0, \\
& \sigma_{z z}=0, \text { for plane stress (CP), } \\
& \sigma_{z z}=v\left(\sigma_{x x}+\sigma_{y y}\right), \text { for plane strain (DP), }
\end{aligned}
$$

with $v$ Poisson ratio.

Let the tensor $\sigma$ and $\mathbf{S}_{\sigma}$ its deviatoric part

$$
\mathbf{S}_{\sigma} \equiv \sigma-\frac{1}{3} \operatorname{tr}(\sigma) 1
$$

In a classical way, the Huber-Von Mises equivalent stress is defined by: $\bar{\sigma} \equiv \kappa\left|\mathbf{S}_{\sigma}\right|$, where $\kappa \equiv \sqrt{\frac{3}{2}}$ a normalisation parameter and $\left|\mathbf{S}_{\sigma}\right| \equiv \sqrt{\operatorname{tr}\left(\mathbf{S}_{\sigma}^{T} \mathbf{S}_{\sigma}\right)}$ the norm of tensor $\mathbf{S}_{\sigma}$.

In order to take into account the asymmetrical behavior between tension and compression in the yield phase transformation surface prediction, a parameter called "Lode parameter" is introduced $[5,6]$.

$$
y_{\sigma} \equiv \frac{6 \kappa}{\left|\mathbf{S}_{\sigma}\right|^{3}} \operatorname{det} \mathbf{S}_{\sigma} .
$$

Now, the problem is to give the equation of the elastic austenitic domain yield surface which is called $F(\sigma)$. In this aim, tools coming from plasticity are used.

As the phase transformation process is independent of the pressure, one suggests to write $F(\sigma)$ as

$$
F(\sigma)=\bar{\sigma} f\left(y_{\sigma}\right)-\sigma_{c}(T)=0 .
$$

The choice of the "shape functions" $f$ is twofold: 
- A simple expression of $f\left(y_{\sigma}\right)$ can be chosen in the form

$$
f\left(y_{\sigma}\right)=1+b y_{\sigma} .
$$

The convexity of $F(\sigma)$ can be assured if and only if $0 \leq b \leq \frac{1}{8}$ [7].

- In reference to the work of Bouvet et al. [8], we have also chosen

$$
f\left(y_{\sigma}\right)=\arccos \left(\frac{1}{3} \arccos \left(1-a\left(1-y_{\sigma}\right)\right)\right),
$$

which ensures that $F(\sigma)$ is convex for $0 \leq a \leq 1$ as also demonstrated by Laydi and Lexcellent [7].

\section{THE ELASTIC STRESS FIELD AROUND A CRACK TIP WITHOUT CURVATURE}

We choose the calculations performed by J. B. Leblond [9] knowing that they have become classics of literature items.

Consider a straight crack in plane strain. Look for the stress field near the crack tip. To do this, define the landmark $\left(O, x_{1}, x_{2}\right)$ with $O$ confused with the tip and $O x_{1}$ located in the extension of the crack, and the corresponding coordinates (Fig. 1).

One obtains classicaly the polar coordinates for mode I (stress intensity factor $K_{I}$ ) plus mode II (stress intensity factor $K_{I I}$ )

$$
\begin{aligned}
\sigma_{r r} & =\frac{K_{I}}{4 \sqrt{2 \pi r}}\left(5 \cos \frac{\theta}{2}-\cos \frac{3 \theta}{2}\right)+\frac{K_{I I}}{4 \sqrt{2 \pi r}}\left(-5 \sin \frac{\theta}{2}+3 \sin \frac{3 \theta}{2}\right), \\
\sigma_{\theta \theta} & =\frac{K_{I}}{4 \sqrt{2 \pi r}}\left(3 \cos \frac{\theta}{2}+\cos \frac{3 \theta}{2}\right)+\frac{K_{I I}}{4 \sqrt{2 \pi r}}\left(-3 \sin \frac{\theta}{2}-3 \sin \frac{3 \theta}{2}\right), \\
\sigma_{r \theta} & =\frac{K_{I}}{4 \sqrt{2 \pi r}}\left(\sin \frac{\theta}{2}+\sin \frac{3 \theta}{2}\right)+\frac{K_{I I}}{4 \sqrt{2 \pi r}}\left(\cos \frac{\theta}{2}+3 \cos \frac{3 \theta}{2}\right),
\end{aligned}
$$

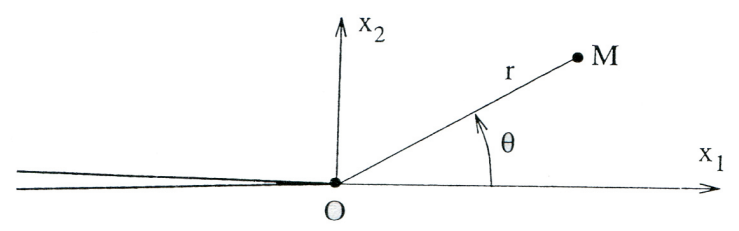

Fig. 1. Crack in plane situation (reference diagram in polar coordinates) [9]

In any case,

$$
\sigma(M(r, \theta))=\chi(r) \mathbf{q}(\theta),
$$

that renders the calculations easy. 
For mode I, the boundary radius expression for phase transformation forward yield surface $(A \Rightarrow M)$ is

$$
r(\theta)=\underbrace{\frac{1}{4 \pi}\left(\frac{K_{t}}{\sigma_{c}}\right)^{2}}_{L_{A m f}}(\underbrace{2 \kappa^{2}\left|\mathbf{S}_{\mathbf{q}}(\theta)\right|^{2}}_{R(\theta)}) f^{2}\left(y_{\sigma}\right) .
$$

Hence, finally, an adimensional term is obtained $r_{L}$

$$
r_{L}(\theta) \equiv \frac{r(\theta)}{L_{A m f}}=R(\theta) f^{2}\left(y_{\sigma}\right),
$$

for

$$
\begin{aligned}
& R(\theta)=3\left|\mathbf{S}_{\mathbf{q}}(\theta)\right|^{2}, \\
& L_{A m f}=\frac{1}{4 \pi}\left(\frac{K_{t}}{\sigma_{c}}\right)^{2} .
\end{aligned}
$$

If one introduces the parameter $\beta$ in order to make the distinction between plane stress $(\mathrm{CP})$ and plane strain (DP).

$$
\beta=\left(\begin{array}{cc}
1 & \text { for } \mathrm{CP} \\
1-2 v & \text { for DP }
\end{array}\right)
$$

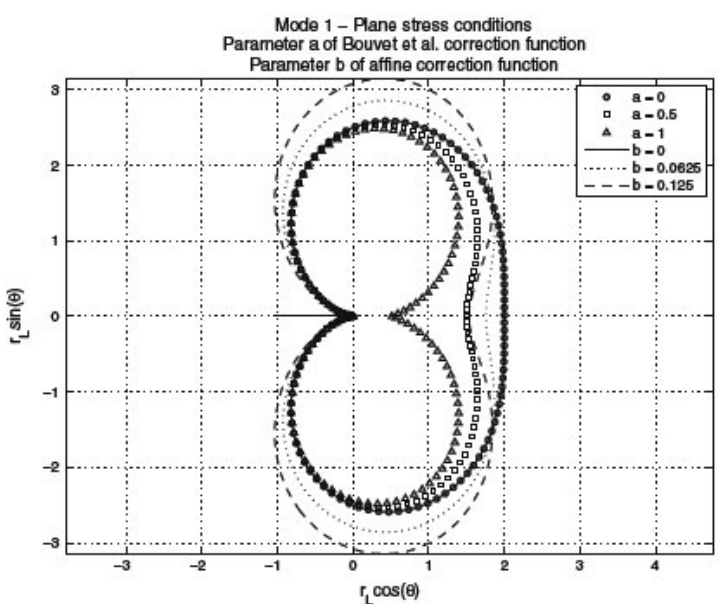

Fig. 2. Mode I - Phase transformation surface for stress plane condition

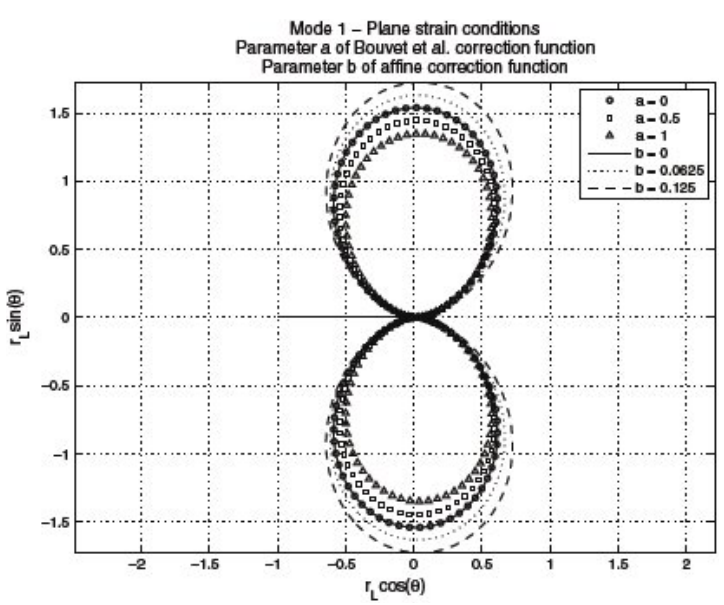

Fig. 3. Mode I - Phase transformation surface for strain plane condition

As it was shown on Figs. 2 and $3, r_{L}(\theta)$ was plotted for the two expressions of $f$ with $\beta=1$ for plane stress condition and $v=0.4$ for plane strain.

- The size of the surfaces is on about twofold in CP than in DP.

- The effect of the asymmetry between tension and compression is more evident in $\mathrm{CP}$ than in DP. 
- The correction with affine function $y_{\sigma}$ provides a field of transformation a bit larger than the function of Bouvet [8].

\section{PROBLEM WITH A CRACK TIP WITH CURVATURE $\sigma$ TO SOLVE}

\subsection{Stress field around the crack tip for mode I}

Let a plate of length $l$, width $w$, thickness $e$ and a fracture length $a$ (Fig. 4). The sample dimensions are chosen to comply with the instructions of the "stress intensity factor handbook" of Murakami et al. [10].

In agreement with Irwin [11], the stress intensity factor $K_{I}$, under the ratio condition $a / w<0.6$ can be written as

$$
K_{I}=g\left(\frac{a}{w}\right) \sigma_{0} \sqrt{\pi a}
$$

with the empirical $g(a / w)$ expression [10]

$$
g\left(\frac{a}{w}\right)=1.122-0.231\left(\frac{a}{w}\right)+10.55\left(\frac{a}{w}\right)^{2}-21.72\left(\frac{a}{w}\right)^{3}+30.39\left(\frac{a}{w}\right)^{4} .
$$

One has to note that an expression of $g$ function integrating the curvature $\rho$ in our knowledge does not exist in the litterature.

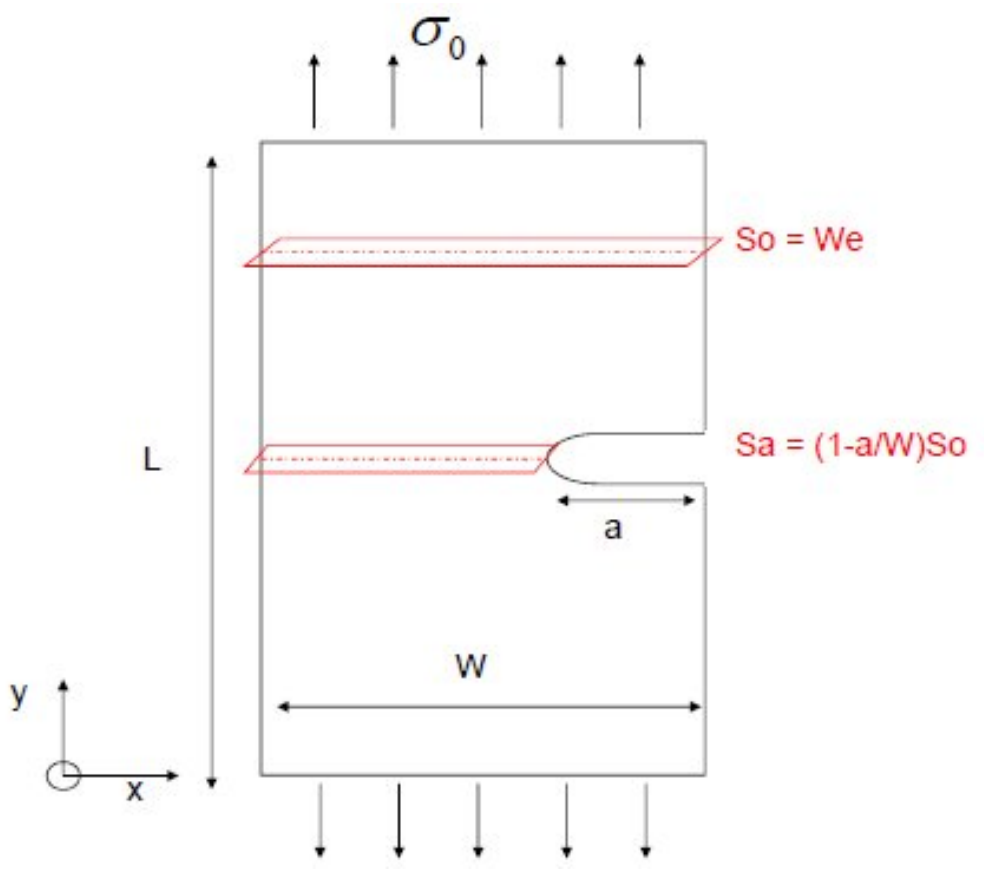

Fig. 4. Standard plate with a crack with curvature $\rho$ under mode I loading 
Starting from the classical stress field around the crack tip for mode I (without curvature radius), Creager and Paris [12] extend the solution for finite curvature $\rho$ as

$$
\begin{aligned}
\sigma_{x x} & =\frac{K_{I}}{\sqrt{2 \pi r}}\left(\cos \frac{\theta}{2}\left(1-\sin \frac{\theta}{2} \sin \frac{3 \theta}{2}\right)-\frac{\rho}{2} \cos \frac{3 \theta}{2}\right), \\
\sigma_{y y} & =\frac{K_{I}}{\sqrt{2 \pi r}}\left(\cos \frac{\theta}{2}\left(1+\sin \frac{\theta}{2} \sin \frac{3 \theta}{2}\right)+\frac{\rho}{2} \cos \frac{3 \theta}{2}\right), \\
\sigma_{x y} & =\frac{K_{I}}{\sqrt{2 \pi r}}\left(\sin \frac{\theta}{2} \cos \frac{\theta}{2} \cos \frac{3 \theta}{2}-\frac{\rho}{2} \sin \frac{3 \theta}{2}\right) .
\end{aligned}
$$

Note again that $r$ has a finite value on the crack with equation $r=\frac{\rho}{2}$ and therefore, the stresses have a finite value, so there is not singularity.

For a given boundary value $\rho_{c}(T)>0$, we seek to calculate the distance $r$

$$
r: \theta \in[0,2 \pi] \rightarrow r(\theta) \geq \frac{1}{2} \rho>0
$$

solution of the non linear equation (4).

The calculations are more complicated because we cannot separate directly $\sigma(M(r, \theta))$ in the product of a $r$ function and a $\theta$ function as we did before for a tip without curvature (Eq. (8)).

\subsection{Huber-von Mises particular case}

One begin with the Huber-Von Mises simple case where $f=1$ in the yield surface equation (4). It means that

$$
\bar{\sigma}=\sigma_{c} .
$$

The tensor $\sigma$ is written as function of an auxiliary variable

$$
z=\frac{\rho}{2 r} .
$$

This delivers the condition on the $z$ variable

$$
0 \leq z \leq 1
$$

and with $\frac{K_{I}}{\sqrt{2 \pi r}}=\frac{K_{i}}{\sqrt{\pi \rho}} \sqrt{z}$ under the shape

$$
\sigma=\frac{K_{i}}{\sqrt{\pi \rho}} \sqrt{z} \Sigma_{\theta}(z) \text { where } \Sigma_{\theta}(z) \equiv \mathbf{A}_{\theta}-z \mathbf{B}_{\theta},
$$

with

$$
\mathbf{A}_{\theta}=\cos \frac{\theta}{2}\left(\begin{array}{ccc}
1-\sin \frac{\theta}{2} \sin \frac{3 \theta}{2} & \sin \frac{\theta}{2} \cos \frac{3 \theta}{2} & 0 \\
\sin \frac{\theta}{2} \cos \frac{3 \theta}{2} & 1+\sin \frac{\theta}{2} \sin \frac{3 \theta}{2} & 0 \\
0 & 0 & 1-\beta
\end{array}\right)
$$


and

$$
\mathbf{B}_{\theta}=\left(\begin{array}{ccc}
\cos \frac{3 \theta}{2} & \sin \frac{3 \theta}{2} & 0 \\
\sin \frac{3 \theta}{2} & -\cos \frac{3 \theta}{2} & 0 \\
0 & 0 & 0
\end{array}\right) .
$$

A simple calculation delivers the eigenvalues of $\mathbf{S}_{\Sigma_{\theta}}$ (deviatoric part of $\Sigma_{\theta}(z)$ )

$$
\lambda_{1}=-2 \frac{\beta}{3} \cos \frac{\theta}{2}, \lambda_{2}=\frac{\beta}{3} \cos \frac{\theta}{2}-\sqrt{z^{2}+\frac{1}{4} \sin ^{2} \theta}, \lambda_{3}=\frac{\beta}{3} \cos \frac{\theta}{2}+\sqrt{z^{2}+\frac{1}{4} \sin ^{2} \theta}
$$

and the Huber-von Mises expression

$$
\bar{\sigma}=\frac{K_{i}}{\sqrt{\pi \rho}} \sqrt{z \Sigma_{\theta}}(z) \text { with } \bar{\Sigma}_{\theta}(z)=3^{\frac{1}{2}} \sqrt{z^{2}+3 p_{\theta}}
$$

where

$$
p_{\theta} \equiv \frac{1}{9}\left(\beta^{2}+3 \sin ^{2} \frac{\theta}{2}\right) \cos ^{2} \frac{\theta}{2} \leqslant \frac{4}{27} \quad \forall \theta .
$$

As a consequence, Eq. (15) is equivalent to

$$
\varphi_{p}(z)=\chi \text { with } \chi=\frac{1}{6} \pi \rho\left(\frac{\sigma_{c}}{K_{I}}\right)^{2},
$$

where $\varphi_{p}$ is defined whatever $p \geq 0$, by

$$
z \in \mathbb{R}^{+} \rightarrow \varphi_{p}(z) \equiv \frac{1}{2} z\left(z^{2}+3 p\right) \in \mathbb{R}^{+}
$$

This function admits as reciprocal one $\psi_{p} \equiv \varphi_{p}^{-1}$ (obtained by the Cardan method) defined on $\mathbb{R}^{+}$by

$$
\psi_{p}(\chi)=\left(\sqrt{\chi^{2}+p^{3}}+\chi\right)^{\frac{1}{3}}-\left(\sqrt{\chi^{2}+p^{3}}-\chi\right)^{\frac{1}{3}} .
$$

Hence by inversion, Eq. (15) admits, whatever $\chi>0$, one solution and only one $z=\frac{\rho}{2 r}$, such that

$$
z=\psi_{p}(\chi)
$$

However, Eq. (14) imposes the condition

$$
0 \leq \psi_{p}(\chi) \leq 1
$$

i.e.

$$
\chi \leqslant \varphi_{p}(z=1)=\frac{1}{2}(1+3 p) \quad \forall p \in\left[0, \frac{4}{27}\right] .
$$

Hence

$$
x \leq \frac{1}{2}
$$

In brief, the following result is established. 
Proposal 1: Eq. (15) admits a unique solution

$$
r=\frac{1}{2} \frac{\varrho}{\psi_{p}(\chi)}
$$

Verifying condition (14) if and only if $\chi$ verifies condition (30).

\subsection{General case}

This case consists in replacing $\sigma_{c}$ by $\sigma_{c} / f\left(y_{\sigma}\right)$ or more simply by $\sigma_{c} / f\left(y_{\Sigma_{\theta}}\right)$ because

$$
\bar{\sigma}=\frac{K_{i}}{\sqrt{\pi \rho}} \sqrt{z \Sigma_{\theta}}(z) \text { and } y_{\sigma}=y_{\Sigma_{\theta}}
$$

i.e.

More precisely, one has the equivalences

$$
\bar{\sigma}=\frac{K_{i}}{\sqrt{\pi \rho}} \sqrt{z \Sigma_{\theta}}(z)=\frac{\sigma_{c}}{f\left(y_{\Sigma_{\theta}}\right)} .
$$

$$
G(\sigma)=\bar{\sigma} f\left(y_{\Sigma_{\theta}}\right)=\sigma_{c} \Leftrightarrow \sqrt{z} G\left(\Sigma_{\theta}\right)=2 \kappa \chi^{\frac{1}{2}} \Leftrightarrow z=\psi\left(\chi \alpha_{\theta}(z)\right),
$$

where $\alpha_{\theta}(z)$ is a continuous function of $z$ and $\theta$ defined by

$$
\alpha_{\theta}(z)=\left|f\left(y_{\Sigma_{\theta}}\right)\right|^{-2} \text {. }
$$

Its explicit expression is obtained easily by combining in the expression (3) the value of $\left|\mathbf{S}_{\Sigma_{\theta}}\right|$

$$
\left|\mathbf{S}_{\Sigma_{\theta}}\right|=\left(\frac{2 \beta^{2}}{3} \cos ^{2} \theta+\frac{\sin ^{2} \theta}{2}+2 z^{2}\right)^{\frac{1}{2}} .
$$

with

$$
\operatorname{det}\left(\mathbf{S}_{\Sigma_{\theta}}\right)=\frac{2 \beta}{3} \cos \frac{\theta}{2}\left(z^{2}+\frac{1}{4} \sin ^{2} \theta-\left|\frac{\beta}{3} \cos \frac{\theta}{2}\right|^{2}\right) .
$$

Moreover, one have the following framing

$$
\frac{1}{8} \eta^{3} \leqslant \chi \alpha_{\theta}(z) \leqslant \frac{1}{2} \eta^{3} \text { and } \eta=\left(6 \chi|f(0)|^{-2}\right)^{\frac{1}{2}} .
$$

This result is the direct consequence of the convexity condition of the $G$ yield function (see Laydi and Lexcellent [13]) which imposes the necessary condition on the $f$ function

$$
3^{-\frac{1}{2}} f(0) \leqslant f(y) \leqslant 2 \times 3^{-\frac{1}{2}} f(0) \quad \forall y \in[-1,1] .
$$

As reference of the single Huber-von Mises problem, the resolution of this problem consist in finding $\widehat{z}$ solution of one of the following equations

$$
\varphi_{p}(\widehat{z})=\chi \alpha_{\theta}(\widehat{z}) \Leftrightarrow \widehat{z}=\psi_{p}\left(\chi \alpha_{\theta}(\widehat{z})\right) \text {. }
$$

Let

$$
\xi \equiv \psi_{\frac{4}{27}}\left(\frac{1}{8} \eta^{3}\right)
$$

Taking into account all the notations and hypothesis, we are able to demonstrate the following theorem. 


\subsection{Application I}

Let the following material parameters

$$
\begin{aligned}
& v=0.3, \rho=0.5(\mathrm{~mm}), K_{I}=50(\mathrm{MPa} \sqrt{\mathrm{m}}) \\
& \sigma_{c}=\left\{\begin{array}{l}
\sigma_{s}=30(\mathrm{Mpa}) \text { start } \\
\sigma_{f}=60(\mathrm{Mpa}) \text { end }
\end{array} \text { stress phase transformation }\right\}
\end{aligned}
$$

Let $f\left(y_{\Sigma}\right)$ an affine function in $y_{\Sigma}$

$$
f\left(y_{\Sigma}\right)=1+b y_{\Sigma}
$$

Laydi and Lexcellent [7] shows that the $G(\Sigma)$ function is convex in $\Sigma$ if $b \in\left[0, \frac{1}{8}\right]$. The verification of the condition need some elementary calculations

$$
\begin{aligned}
\eta & =\left(6 \chi|f(0)|^{-2}\right)^{\frac{1}{2}}=\left(\pi|f(0)|^{-2} \rho\left(\frac{\sigma_{c}}{K_{I}}\right)^{2}|f(0)|^{-2}\right)^{\frac{1}{2}} \\
& =\left\{\begin{array}{l}
0.08 \text { start phase transformation } \\
0.13 \text { end phase transformation }
\end{array}\right\}<0.34 .
\end{aligned}
$$

In the two cases, the condition of unicity of the solution is verified. The zones of phase transformation for plane stress (CP) and plane strain (DP) conditions are shown as function of the $b$ parameter on Fig. 5 .
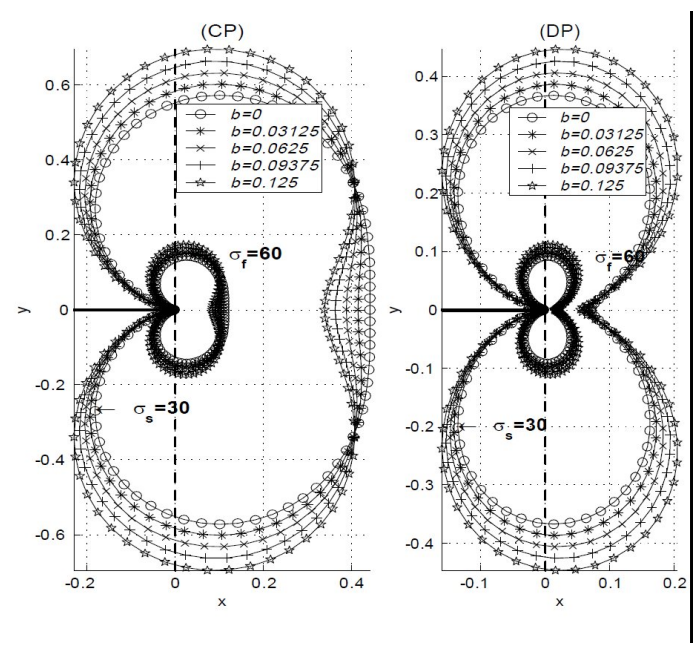

Fig. 5. Cracked plate with curvature under mode I (Application I)
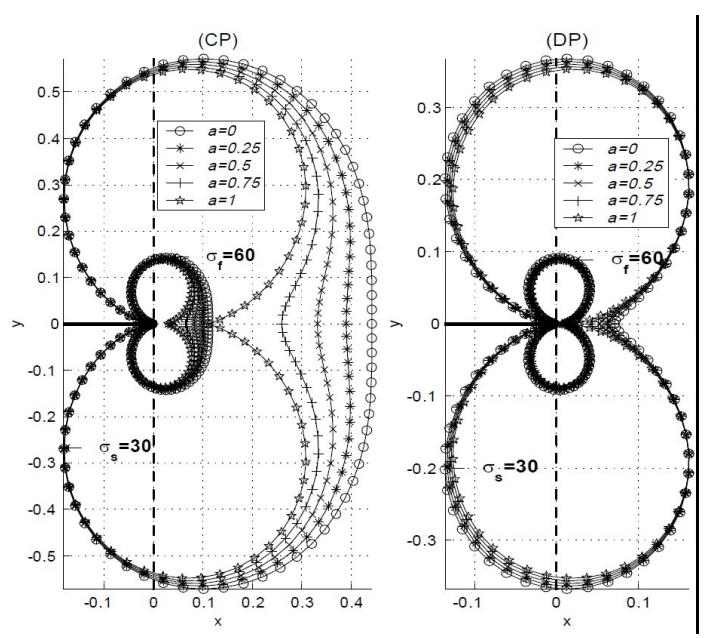

Fig. 6. Cracked plate with curvature under mode I (Application II) 


\subsection{Application II}

Let

$$
f\left(y_{\Sigma}\right)=\cos \left(\frac{1}{3} \arccos \left(1-\alpha\left(1-y_{\Sigma}\right)\right)\right) .
$$

A new time, Laydi and Lexcellent [7] have shown that $G(\Sigma)$ is convex if $a \in[0,1]$.

The condition of convexity is verified whatever a belonging to $[0,1]$. The zones of phase transformation for plane stress (CP) and plane strain (DP) conditions are shown as function of the $b$ parameter on Fig. 2. The zones of phase transformation for plane stress (CP) and plane strain (DP) conditions are shown as function of the $a$ parameter on Fig. 6 .

\section{COMPARISON BETWEEN EXPERIMENTS AND PREDICTIONS}

One has to underline that there is not important difference in the phase transformation zones exhibited for the two chosen shape functions e.g. "affine" (Fig. 2) or "arcos" one (Fig. 3) .The impact of the parameters of assymmetry $b$ and $a$ is negligible in plane strain and effective in plane stress. Moreover, coupled thermomechanical problems can be solved by digital image correlation (DIC). Thus Daly et al. [1] measured by DIC deformations around the crack tip on nitinol thin plates. This "in situ" optical method provides information about the phase transformation zones by locating deformations. The choice of the "Linear Elastic Fracture Mechanics" (LEFM) hypothesis seems justified by the small size of the transformation zones as shown on Fig. 7.

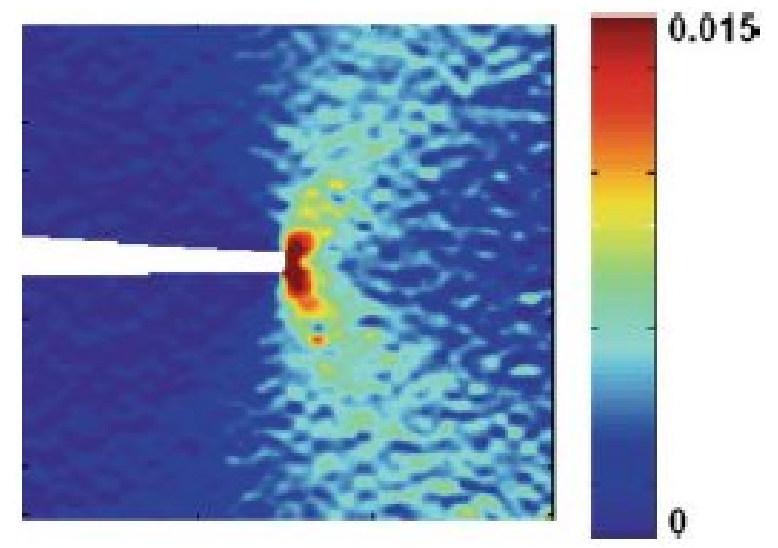

Fig. 7. Measurement of $\varepsilon_{y y}$ obtained by DIC at the crack tip for $K_{I}=44 \mathrm{Mpa} \sqrt{\mathrm{m}}[1]$

The shape of the lobes of the transformation surface inclined at around $60^{\circ}$, validates the taking into account the assymmetry between tension and compression in the calculations.

Tensile tests have been performed in the SYMME laboratory of Annecy on samples exhibiting different radii of curvature [14].

Depending on the dimensions of the sample and on the curvature radius, we can calculate the stress intensity factors by making use of correction cefficient from Eq. (12). 


$$
\begin{array}{ccccc}
\rho(\mathrm{mm}) & 0.25 & 0.5 & 1 & 1.5 \\
K_{i c}(\mathrm{Mpa} \sqrt{\mathrm{m}}) & 91 & 102 & 108 & 115
\end{array}
$$

Its means that the intensity factor $K_{I c}$ is slighty dependant of the curvature radius $\rho$.

The shape of the transformation zones shown on Fig. 8 for plane stress conditions is consistent with our predictions.

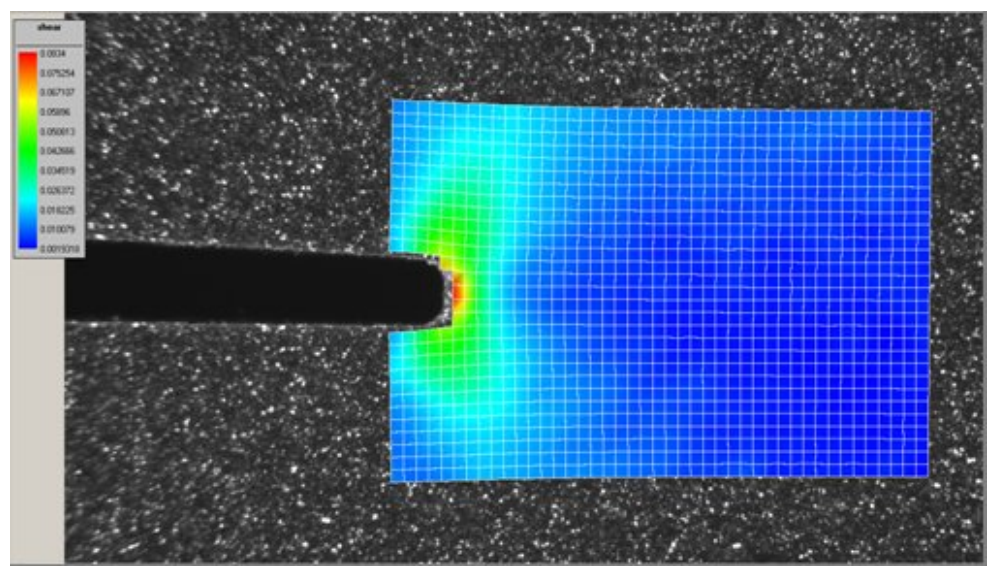

Fig. 8. Overall photo of a plate with a curvature radius under mechanical loading (FML) [14]

\section{CONCLUSION}

Comparison between experiments and predictions for mode I shows us a qualitative agreement for crack tip with curvature.

Figs. 1 and 2 on one part and 4 and 5 on an another part show that the extend of the phase transformation zone is seriously greater for tip without curvature than with curvature. In other words, note the difference in severity between an angular defect (zero radius at the crack tip) and a regular defect (finite radius of curvature). The difference between stress concentration and stress singularity can be used to remove the stress singularity near an angular defect. In order to stop the progress, simply "rounding" the tip piercing in a circular hole (process known as SNCF method! (note : SNCF corrresponds to french railways company) [15]. Moreover, crack tip stress-induced martensitic transformation and the resulting stress distribution in a Ni-Ti have been also analysed by Maletta et al. [16].Their recent analytical model on SMA pseudoelasticity occurring in the crack tip region "causes a complex and unsual stress distribution" [17].It means that the problem to solve is complex. The transition of phase transformation mechanisms at microscale to macroscale is not entirely understood.

\section{REFERENCES}

[1] S. Daly, A. Miller, G. Ravichandran, and K. Bhattacharya. An experimental investigation of crack initiation in thin sheets of nitinol. Acta Materialia, 55, (18), (2007), pp. 6322-6330. doi:10.1016/j.actamat.2007.07.038. 
[2] S. Yi and S. Gao. Fracture toughening mechanism of shape memory alloys due to martensite transformation. International Journal of Solids and Structures, 37, (38), (2000), pp. 5315-5327. doi:10.1016/s0020-7683(99)00213-9.

[3] X. M. Wang, Y. F. Wang, A. Baruj, G. Eggeler, and Z. F. Yue. On the formation of martensite in front of cracks in pseudoelastic shape memory alloys. Materials Science and Engineering: $A$, 394, (1), (2005), pp. 393-398. doi:10.1016/j.msea.2004.11.029.

[4] S. W. Robertson and R. O. Ritchie. In vitro fatigue-crack growth and fracture toughness behavior of thin-walled superelastic Nitinol tube for endovascular stents: A basis for defining the effect of crack-like defects. Biomaterials, 28, (4), (2007), pp. 700-709. doi:10.1016/j.biomaterials.2006.09.034.

[5] L. Orgéas and D. Favier. Stress-induced martensitic transformation of a NiTi alloy in isothermal shear, tension and compression. Acta Materialia, 46, (15), (1998), pp. 5579-5591. doi:10.1016/s1359-6454(98)00167-0.

[6] P. Vacher and C. Lexcellent. Study of pseudo-elastic behavior of polycristalline shape memory alloys by resistivity measurements and acoustic emission. In Proceedings of ICM VI, Kyoto, Japan, (1991).

[7] M. R. Laydi and C. Lexcellent. Yield criteria for shape memory materials: convexity conditions and surface transport. Mathematics and Mechanics of Solids, 15, (2), (2010), pp. 165-208. doi:10.1177/1081286508095324.

[8] C. Bouvet, S. Calloch, and C. Lexcellent. Mechanical behavior of a Cu-Al-Be shape memory alloy under multiaxial proportional and nonproportional loadings. Journal of Engineering Materials and Technology, 124, (2), (2002), pp. 112-124. doi:10.1115/1.1448324.

[9] J. B. Leblond and P. Germain. Mécanique de la rupture fragile et ductile. Hermés-Lavoisier, (2003).

[10] Y. Murakami. Stress intensity factor handbook. The Society of Material Science, Pergamon Press, (1987).

[11] G. R. Irwin. Analysis of stresses and strains near the end of a crack traversing a plate. Journal of Applied Mechanics, 24, (3), (1957), pp. 361-364.

[12] M. Creager and P. C. Paris. Elastic field equations for blunt cracks with reference to stress corrosion cracking. International Journal of Fracture, 3, (4), (1967), pp. 247-252. doi:10.1007/bf00182890.

[13] M. R. Laydi and C. Lexcellent. Rice local phase angle study for a delamination problem between a shape memory alloy and an elastic material. Archive for Rational Mechanics and Analysis, 204, (3), (2012), pp. 977-1007. doi:10.1007/s00205-012-0495-6.

[14] V. Taillebot. Contribution à l'étude de la rupture desalliages à mémoire de forme. PhD thesis, Universite de Franche Comte, (2012).

[15] P. Suquet. Rupture et plasticité. In Ecole Polytechnique, (2003).

[16] C. Maletta, E. Sgambitterra, and F. Furgiuele. Crack tip stress distribution and stress intensity factor in shape memory alloys. Fatigue E Fracture of Engineering Materials $\mathcal{E}$ Structures, 36, (9), (2013), pp. 903-912. doi:10.1111/ffe.12055.

[17] C. Maletta and F. Furgiuele. Fracture control parameters for NiTi based shape memory alloys. International Journal of Solids and Structures, 48, (11), (2011), pp. 1658-1664. doi:10.1016/j.ijsolstr.2011.02.014. 